\title{
Basal bodies in Xenopus
}

\section{(0) 0 A A cess

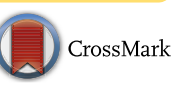

Siwei Zhang and Brian J. Mitchell

\begin{abstract}
Xenopus has been one of the earliest and most important vertebrate model organisms for investigating the role and structure of basal bodies. Early transmission electron microscopy studies in Xenopus revealed the fine structures of Xenopus basal bodies and their accessory structures. Subsequent investigations using multiciliated cells in the Xenopus epidermis have further revealed many important features regarding the transcriptional regulation of basal body amplification as well as the regulation of basal body/cilia polarity. Future basal body research using Xenopus is expected to focus on the application of modern genome editing techniques (CRISPR/TALEN) to characterize the components of basal body proteins and their molecular functions.
\end{abstract}

Keywords: Xenopus, Basal body, Centriole, Deuterosome, Ciliogenesis, Cilia, Multiciliated cells

\section{The organism}

The term Xenopus refers to a collection of approximately 20 fully aquatic frog species within the genus Xenopus. In scientific classifications, they belong to Kingdom Animalia, Phylum Chordata, Class Amphibia, Order Anura, and Family Pipidae. The early Xenopus embryo, due to their large size and free development outside the mother's body, have been one of the most important models for the investigation of early vertebrate development as well as basic biology for many years [1]. Initial investigations using the Xenopus species, which can be dated back to the mid-late 1800 s, are mainly restricted to the larger, easy-to-handle $X$. laevis strain. However, a genome duplication event during the evolution of $X$. laevis has been discovered, which indicates that $X$. laevis is a pseudotetraploid species with genetic redundancy [2]. This has prevented detailed genetic studies to be performed on the Xenopus species. However, a diploid Xenopus system $X$. (Silurana) tropicalis with a much smaller genome size was introduced allowing detailed genetic manipulations to be performed in Xenopus [3, 4]. Recently, the full genome sequence of both $X$. laevis and X. tropicalis has been released to provide the basis for advanced genomic manipulations, such as CRISPR/Cas9 and/or TALEN,

*Correspondence: brian-mitchell@northwestern.edu

Department of Cell and Molecular Biology, Feinberg School of Medicine, Northwestern University, Chicago, IL 60611, USA in addition to the traditional morpholino oligo (MO) knockdown approach [5].

\section{Basic basal body structure}

The basal body is a specialized form of centriole that is located at the cell membrane and provides the anchoring point as well as the growth basis for the cilium. As with most of the organisms that possess centrioles during their cell cycle, the main structure of the Xenopus basal body is the characteristic 9 blades of microtubule triplets [6, 7]. Xenopus has proven to be an extremely powerful system for the analysis of cilia formation and function [6]. Unfortunately, relative to many other model organisms, there is a paucity of transmission electron microscopy (TEM)-level detail of structural information, particularly for the basal body. Despite the fact that most internal organs possess primary cilium, our literature search found no structural information regarding the basal bodies of these cilia. Likewise, the gastrocoel roof plate (GRP) in Xenopus is an analogous structure to the mouse embryonic node (or Kupffer's vesicle in zebrafish), and it possesses motile mono-cilia which generate the directional flow that establishes embryonic left-right patterning [7]. Again, no structural detail of these basal bodies and cilia is currently available. However, it is reasonable to assume that, similar to what has been found in other organisms, the structure of the ciliary axoneme of immotile and motile mono-cilia differs significantly. Another special case that is worth 
mentioning is the outer segments (OS) of both rod and cone photoreceptors found in Xenopus retina, which arise as an elaboration of an immotile primary cilia. In mature Xenopus retina, this modified ciliary structure remains as the sole cytoplasmic connection that bridges the inner segment and OS of the photoreceptors [8]. Unfortunately, no ultrastructural detail of this ciliary axoneme is available. Perhaps the most well-studied cilia in Xenopus are the motile cilia present in the multiciliated cells (MCCs) that occur on the surface of the early embryo (Fig. 1). Each MCC possesses approximately 150 basal bodies that will nucleate their motile cilia. For the purposes of this primer, we will restrict our discussion to this particular form of basal body.

The basal bodies of Xenopus MCCs can be labeled by the strong presence of $\gamma$-tubulin during both live imaging and immunostaining [9]. Several additional tubulin genes, in addition to the $\alpha-, \beta$-, and $\gamma$-tubulin genes found in most organisms, have been identified in the Xenopus system. The $\delta$-tubulin gene (tubd1, tubulin, delta 1) gene has been identified in X. laevis by the Stearns lab [10].
The $\varepsilon$-tubulin (tube1, tubulin, epsilon 1) gene has also been identified in $X$. laevis for its roles in centriole duplication and microtubule organization $[11,12]$. In addition, a special tubulin gene, $\zeta$-tubulin (tubz1, tubulin, zeta 1), has also been characterized as an important component of the basal foot in MCCs. In contrast, in cycling cells, $\zeta$-tubulin does not locate to centrioles but rather associates with the TRiC/CCT cytoplasmic chaperone complex in the cytoplasm [13]. Interestingly, from an evolutionary point of view, the latter three tubulin families form a coconserved module, named the ZED module. This ZED module has been independently lost in several branches of the evolution tree, such as in higher fungi, higher plants, and placenta mammals. It is also important to note that for the species that possess the ZED module, $\varepsilon$-tubulin gene is always present, while there is a chance of losing either $\delta$ - or $\zeta$-tubulin, but not both [13]. It has been proposed that the presence of the ZED module may be essential for the formation of centriolar appendages; however, further investigations will be required to resolve this question.
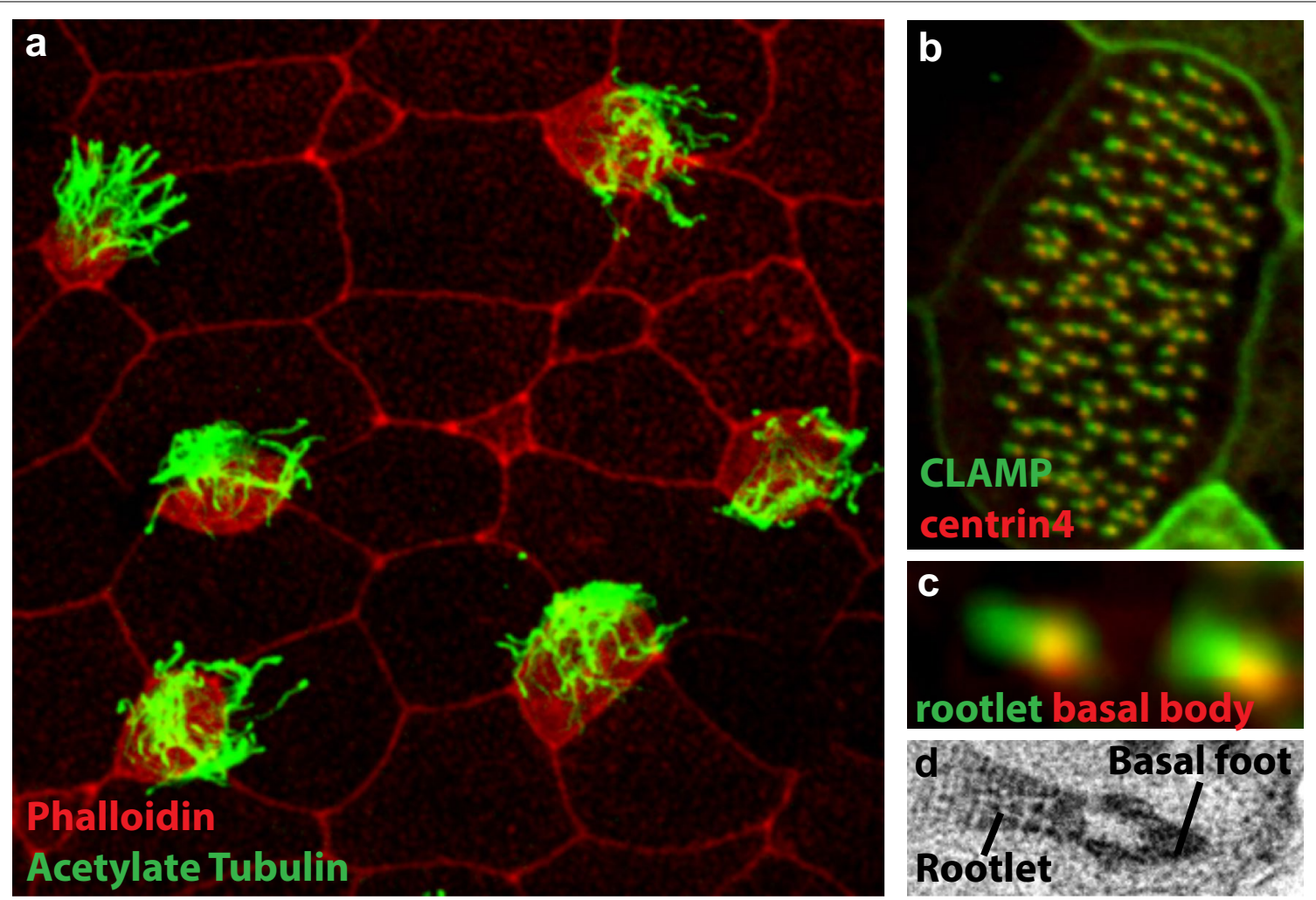

Fig. 1 Multiciliated epithelium of Xenopus. a The punctate pattern of MCCs in the skin of Xenopus embryos with cilia marked with anti-acetylated tubulin (green) and cell boundaries marked with phalloidin (red). b Single MCC with the basal bodies marked with Centrin4-RFP (red) and the rootlets marked with GFP-CLAMP (green). c Close-up image of two basal body/rootlet pairs from (b). d TEM image showing the rootlet and the basal foot in opposite orientation surrounding the basal body. In all images, anterior is to the left and posterior is to the right. The effective stroke of the ciliary beat is oriented to the posterior 


\section{Additional basal body structures or accessory structures}

The systematic study of Xenopus basal bodies and their accessory structures first started in the late 1960s [14]. Similar to other vertebrate species, there are transition fibers, whose structure is similar to distal appendages, that help anchor the basal body to the membrane in the transition zone (Fig. 2, inlay) [15]. Most cells that possess an immotile primary cilium are characterized by the association of a daughter centriole positioned orthogonal to the basal body throughout the lifetime of the cilia [16]. In contrast, in MCCs, the basal bodies of motile cilia are free-standing structures with no associated daughter centrioles $[17,18]$. During maturation, centrioles acquire appendages such as transitional fibers and basal feet that serve as anchoring structures to stabilize the basal bodies $[19,20]$. In mature, polarized MCCs, the basal foot projects posteriorly orthogonal to the basal body in the direction of the ciliary effective stroke (Fig. 2, inlay). Both $\zeta$ - and $\varepsilon$-tubulin have been shown to localize at the basal foot in Xenopus, and the foot serves as a microtubule organizing center (MTOC) [13, 21-23]. In contrast, another accessory structure, the striated rootlet, projects anteriorly away from the basal body and is always positioned $180^{\circ}$ opposite from the basal foot (Fig. 1). The rootlet is a large striated, fibrous structure that extends from the basal body into the cytoplasm and provides structural support to the cilium, as well as demarcating the orientation of motile cilium. This rootlet is prominent in many TEM images of MCC basal bodies and can be visualized using light microscopy based on the localization of rootlet associated proteins such as Mig12 and Spef1/CLAMP (Figs. 1b-d, 2) [24, 25]. The structure of the rootlet is complex, sometimes containing multiple branches (Fig. 2) [14-26]. While the rootlet is generally thought to serve as a mechanical anchor for the beating cilium, it is possible that it also provides a scaffold for signaling events. The Wnt/PCP signaling molecule Dvl2 localizes adjacent to the centriole along the rootlet [24]. Similarly, several focal adhesion proteins including FAK, Vinculin, and Paxillin associate with the proximal and distal ends of the rootlet and are likely to provide certain tension based signaling [27]. Finally, while the basal foot associates primarily with microtubules, the rootlet appears to interact with the dense sub-apical actin network surrounding these cilia [21].

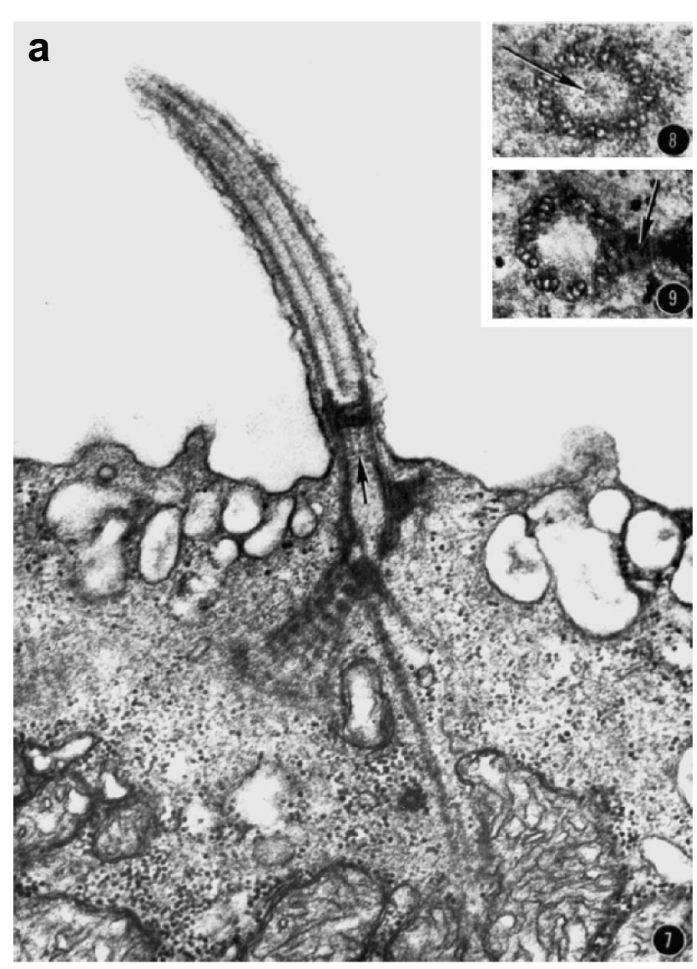

b
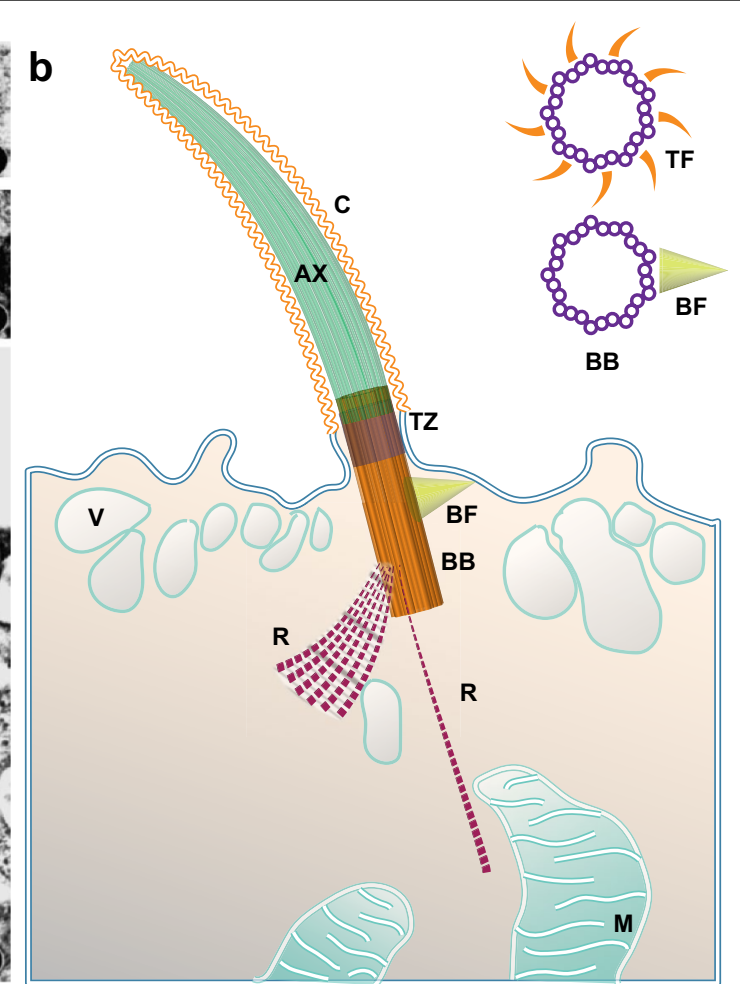

Fig. 2 Vertical cross section of a Xenopus motile cilium. a TEM image of a single cilium with drawing representation of individual structures $\mathbf{b} A X$ axoneme, $B B$ basal body, $B F$ basal foot, $C$ cilium, $M$ mitochondria, $R$ rootlet, $T Z$ transition zone, $T F$ transition fibers, $V$ vesicles. In the figure, anterior is to the left and posterior is to the right. Image in a was taken with permission from Steinmann 1968 


\section{The origins of basal body}

The basal bodies in Xenopus are both converted from centrioles as well as built de novo depending on the cell type. For cells that generate a single cilium, such as cells found in the GRP and gut, basal bodies are converted from the mother centriole similar to other systems [28]. While cycling cells contain both a mother and a daughter centriole, typically it is only the older "mother" centriole that has gone through a full cell cycle that is competent to become a basal body. Interestingly, ectopic over-expression of Foxj1, a protein that plays a crucial role during the differentiation and maintenance of ciliated cells, is able to drive basal body conversion inducing the formation of 1-2 cilia per cell when expressed in non-ciliated epithelial cells [29]. This phenomenon suggests that, in the Xenopus skin, both the mother and daughter centrioles may maintain a certain level of basal body competency. In MCCs that generate dozens of basal bodies, the process appears to be quite distinct. Instead of nucleating from an older "mother" centriole, the vast majority of basal bodies nucleate from a structure termed the deuterosome [30]. The regulation of this process is still poorly understood but clearly requires the key centriole duplication regulating proteins Plk4 and Cep152 [30, 31]. Remarkably, while these cells are postmitotic, the nascent centrioles are immediately competent to become cilia-nucleating basal bodies without going through a cell cycle. As soon as centrioles are generated, they begin their migration to the apical cell surface and immediately initiate cilia formation. How this centriole-to-basal body conversion is regulated remains a mystery.

While the structures of centrioles and basal bodies are generally comparable, there are important structural and functional distinctions between them. For example, microtubules (part of the ciliary axoneme) directly and specifically emerge from the distal end of the basal body, whereas cytoplasmic and mitotic microtubules nucleate in all directions from the pericentriolar material surrounding the centriolar pair of the centrosome. In addition to the basal body-specific appendages detailed above, another important distinction between centrioles and basal bodies is their relationships with cell membranes. Basal bodies associate with membrane-bound vesicles as the vesicles migrate to and fuse with the apical cell membrane [24]. This membrane association is critical to basal body function and components of the basal body-linked transition zone and is thought to regulate distinct membrane compartments. More detailed reviews on this topic, including the structural and functional differences between ciliary membrane and cell membrane, are provided in $[32,33]$.

\section{The life cycle of basal body and its other functions}

Most cells in Xenopus contain a centriolar pair, or centrosome that functions as the MTOC and is critical during mitosis. Xenopus do not possess basal bodies through all stages of their life cycle. During early development when cells are rapidly dividing they do not possess primary cilium during interphase. In fact, cells with primary cilia have not been widely described and have only been reported in the GRP, the neural tube, and later during organogenesis (Fig. 3) [7, 29, 34-39]. Most of the early embryonic cells do not have cilia, and the formation of basal bodies occurs at distinct times in different tissues (Fig. 3). The massive centriole amplification which occurs in MCCs begins around stages $16-17$, and these cells typically complete ciliogenesis between stage 20 and 22. Prior to becoming basal bodies and forming cilia, the numerous centrioles of MCCs are essential for nucleating a pool of acetylated stable microtubules that is important for the process of radial intercalation, by which MCCs insert into the outer epithelium [40].

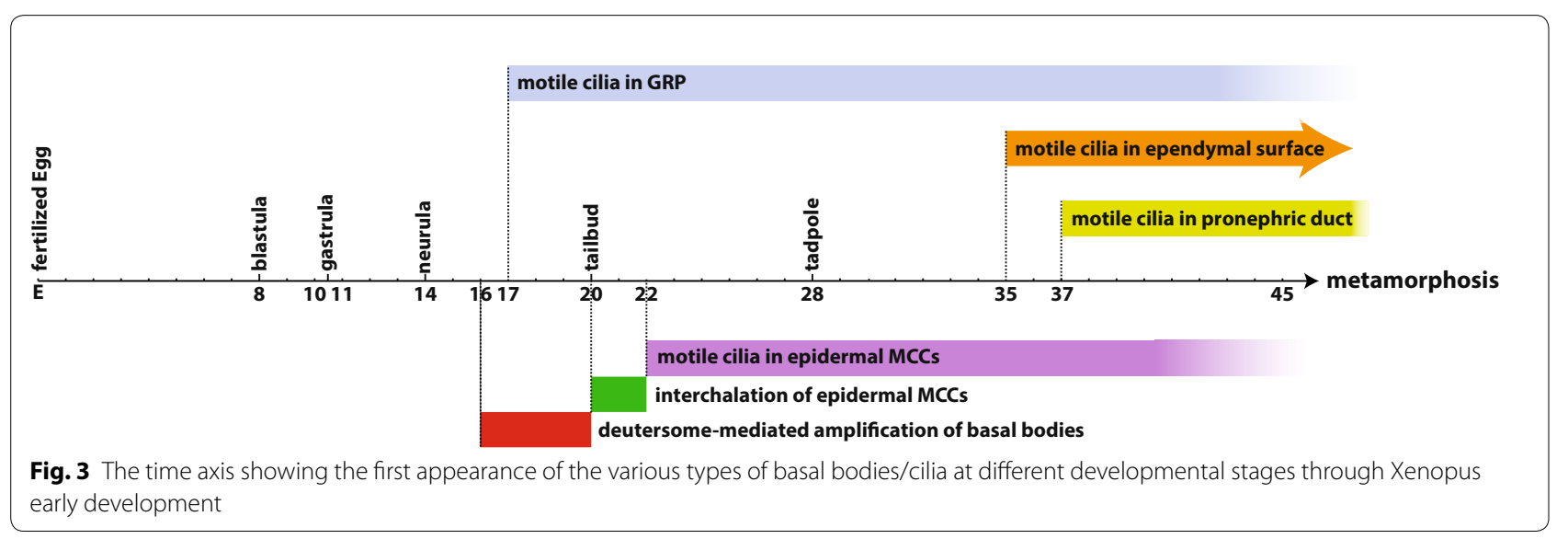




\section{Identification of basal body components}

To date, no studies that systematically address the protein components of Xenopus basal bodies have been identified. However, numerous proteins are known to localize to basal bodies, including many proteins that localize to centrioles in other systems, and the components seem quite comparable to other vertebrate (and non-vertebrate) centrioles. In addition, gene expression analyses in MCCs indicate the up-regulation of many centriolar components that are most certainly contained in the basal bodies of motile cilia [29, 34, 41-43]. While many centriolar components (e.g., Centrin, Poc1, HYLS, Sas6, Plk4, Cep152, and numerous others) appear similar to all centrioles, other components (e.g., Dvl2) likely represent unique features of multiciliated basal bodies.

\section{Notable basal body findings}

The ciliated epithelium of Xenopus has proven to be a particularly powerful system for the study of cilia and basal bodies (reviewed in [6, 44]). Specifically, this system has provided the first evidence of PCP signaling and fluid flow affecting cilia/basal body polarity [24, 25, 45], the first evidence of a septin-based cilia diffusion barrier [46], the characterization of MCC-specific transcriptional regulators [29, $41-43,47]$, the first characterization of miRNA-mediated regulation of basal body duplication and ciliogenesis [48, 49], and the first molecular characterization of the basal body-generating structure the deuterosome [30]. These and many other important discoveries were facilitated by the molecular, embryological, and imaging techniques that are available in Xenopus coupled with the fact that the ciliated epithelia develop on the external surface of the embryos rather than inside the organism. Notably, the discoveries in Xenopus have been validated in other vertebrate systems [50-53]. In addition, many human genetic defects have been authenticated and more thoroughly characterized using the tools available in Xenopus [54-56].

\section{Strengths and future of basal body research in Xenopus}

It is a very exciting time to be using Xenopus as a model system to study basal bodies. Recent advances in the detailed quantification of both protein and RNA levels across early Xenopus development stages promises to facilitate the analysis of many developmental processes including basal body formation and functions [57]. In addition, recent advances in genome editing technologies including TALENs and CRISPR/Cas hold great potential to allow rapid analysis of genetic mutations [54]. Specifically, the ability to couple CRISPR/Cas with homologous recombination to insert either fluorescent markers or specific mutations will greatly enhance our ability to model human disease in Xenopus. Important questions that remain to be answered are as follows: how are centriole amplification and centriole-to-basal body conversion regulated in MCCs that are no longer progressing through the cell cycle; what is the driving force of apical migration/insertion of basal bodies; how do basal bodies and their accessory structures interact with the cytoskeleton as well as with the cell cortex; and what are the similarities/differences between the basal bodies of motile and primary cilia. With these new tools in hand, the next few years will certainly lead to many new advances in our understanding of basal body formation and functions.

\section{Abbreviations}

TEM: transmission electron microscopy; MCC: multiciliated cell; MO: morpholino oligo; GRP: gastrocoel roof plate; OS: outer segments; MTOC: microtubule organizing center; PCP: planar cell polarity.

\section{Authors' contributions}

SZ and BJM contributed to writing this review. Both authors read and approved the final manuscript.

\section{Acknowledgements}

We would like to thank Sun Kim and Jennifer Mitchell for helpful comments on the manuscript. BJM was supported by NIH-NIMGS 2R01GM089970.

\section{Competing interests}

The authors declare that they have no competing interests.

Received: 13 November 2015 Accepted: 20 January 2016

Published online: 03 February 2016

\section{References}

1. Harland RM, Grainger RM. Xenopus research: metamorphosed by genetics and genomics. Trends Genet. 2011;27(12):507-15.

2. Cannatella DC, Desa RO. Xenopus laevis as a model organism. Syst Biol. 1993;42(4):476-507

3. Thiebaud CH, Fischberg M. DNA content in genus Xenopus. Chromosoma. 1977;59(3):253-7.

4. Tymowska J. Karyotype analysis of Xenopus tropicalis Gray, Pipidae. Cytogenet Cell Genet. 1973;12(5):297-304.

5. Hellsten U, Harland RM, Gilchrist MJ, Hendrix D, Jurka J, Kapitonov V, et al. The genome of the Western clawed frog Xenopus tropicalis. Science. 2010;328(5978):633-6.

6. Werner ME, Mitchell BJ. Understanding ciliated epithelia: the power of Xenopus. Genesis. 2011. PubMed PMID: 22083727

7. Schweickert A, Weber T, Beyer T, Vick P, Bogusch S, Feistel K, et al. Cilia-driven leftward flow determines laterality in Xenopus. Curr Biol. 2007;17(1):60-6.

8. Eckmiller MS. Renewal of the ciliary axoneme in cone outer segments of the retina of Xenopus laevis. Cell Tissue Res. 1996;285(1):165-9.

9. Felix MA, Antony C, Wright M, Maro B. Centrosome assembly in vitro: role of gamma-tubulin recruitment in Xenopus sperm aster formation. J Cell Biol. 1994;124(1-2):19-31.

10. Piard-Ruster K, Stearns T. Unpublished AAL274501. 2001. PubMed PMID: AAL27450.1

11. Chang P, Giddings TH Jr, Winey M, Stearns T. Epsilon-tubulin is required for centriole duplication and microtubule organization. Nat Cell Biol. 2003;5(1):71-6.

12. Chang P, Stearns T. Delta-tubulin and epsilon-tubulin: two new human centrosomal tubulins reveal new aspects of centrosome structure and function. Nat Cell Biol. 2000;2(1):30-5.

13. Turk E, Wills AA, Kwon T, Sedzinski J, Wallingford JB, Stearns T. Zeta-tubulin is a member of a conserved tubulin module and is a component of the centriolar basal foot in multiciliated cells. Curr Biol. 2015;25(16):2177-83. 
14. Steinman RM. An electron microscopic study of ciliogenesis in developing epidermis and trachea in the embryo of Xenopus laevis. Am J Anat. 1968;122(1):19-55.

15. Anderson RG. The three-dimensional structure of the basal body from the rhesus monkey oviduct. J Cell Biol. 1972;54(2):246-65.

16. Alieva IB, Vorobjev IA. Vertebrate primary cilia: a sensory part of centrosomal complex in tissue cells, but a "sleeping beauty" in cultured cells? Cell Biol Int. 2004;28(2):139-50

17. Abughrien BM, Dore MA. Ciliogenesis in the uterine tube of control and superovulated heifers. Cells Tissues Organs. 2000;166(4):338-48.

18. Anderson RG, Brenner RM. The formation of basal bodies (centrioles) in the Rhesus monkey oviduct. J Cell Biol. 1971;50(1):10-34.

19. Dawe HR, Farr H, Gull K. Centriole/basal body morphogenesis and migration during ciliogenesis in animal cells. J Cell Sci. 2007;120(Pt 1):7-15.

20. Rosenbaum JL, Witman GB. Intraflagellar transport. Nat Rev Mol Cell Biol. 2002;3(11):813-25.

21. Werner ME, Hwang P, Huisman F, Taborek P, Yu CC, Mitchell BJ. Actin and microtubules drive differential aspects of planar cell polarity in multiciliated cells. J Cell Biol. 2011;195(1):19-26.

22. Gordon RE. Three-dimensional organization of microtubules and microfilaments of the basal body apparatus of ciliated respiratory epithelium. Cell Motil. 1982;2(4):385-91.

23. Sandoz D, Chailley B, Boisvieux-Ulrich E, Lemullois M, Laine MC, BautistaHarris G. Organization and functions of cytoskeleton in metazoan ciliated cells. Biol Cell. 1988;63(2):183-93.

24. Park TJ, Mitchell BJ, Abitua PB, Kintner C, Wallingford JB. Dishevelled controls apical docking and planar polarization of basal bodies in ciliated epithelial cells. Nat Genet. 2008;40(7):871-9.

25. Mitchell B, Stubbs JL, Huisman F, Taborek P, Yu C, Kintner C. The PCP pathway instructs the planar orientation of ciliated cells in the Xenopus larval skin. Curr Biol. 2009;19(11):924-9.

26. Chien YH, Werner ME, Stubbs J, Joens MS, Li J, Chien S, et al. Bbof1 is required to maintain cilia orientation. Development. 2013;140(16):3468-77

27. Antoniades I, Stylianou P, Skourides PA. Making the connection: ciliary adhesion complexes anchor basal bodies to the actin cytoskeleton. Dev Cell. 2014;28(1):70-80.

28. Kobayashi T, Dynlacht BD. Regulating the transition from centriole to basal body. J Cell Biol. 2011;193(3):435-44.

29. Stubbs JL, Oishi I, Izpisua Belmonte JC, Kintner C. The forkhead protein Foxj1 specifies node-like cilia in Xenopus and zebrafish embryos. Nat Genet. 2008;40(12):1454-60.

30. Klos Dehring DA, Vladar EK, Werner ME, Mitchell JW, Hwang P, Mitchell BJ. Deuterosome-mediated centriole biogenesis. Dev Cell. 2013;27(1):103-12.

31. Wong YL, Anzola JV, Davis RL, Yoon M, Motamedi A, Kroll A, et al. Cell biology. Reversible centriole depletion with an inhibitor of Polo-like kinase 4. Science. 2015;348(6239):1155-60.

32. Reiter JF, Blacque OE, Leroux MR. The base of the cilium: roles for transition fibres and the transition zone in ciliary formation, maintenance and compartmentalization. EMBO Rep. 2012;13(7):608-18.

33. Ishikawa H, Marshall WF. Ciliogenesis: building the cell's antenna. Nat Rev Mol Cell Biol. 2011;12(4):222-34

34. Chung MI, Peyrot SM, LeBoeuf S, Park TJ, McGary KL, Marcotte EM, et al. RFX2 is broadly required for ciliogenesis during vertebrate development. Dev Biol. 2012;363(1):155-65.

35. Zhang B, Tran U, Wessely O. Expression of Wht signaling components during Xenopus pronephros development. PloS One. 2011;6(10):e26533.

36. Whitehead JL, Wang SY, Bost-Usinger L, Hoang E, Frazer KA, Burnside B. Photoreceptor localization of the KIF3A and KIF3B subunits of the heterotrimeric microtubule motor kinesin II in vertebrate retina. Exp Eye Res. 1999;69(5):491-503.
37. McGrath J, Brueckner M. Cilia are at the heart of vertebrate left-right asymmetry. Curr Opin Genet Dev. 2003;13(4):385-92.

38. Brandli AW. Towards a molecular anatomy of the Xenopus pronephric kidney. Int J Dev Biol. 1999;43(5):381-95.

39. Hagenlocher C, Walentek P, Muller C, Thumberger T, Feistel K. Ciliogenesis and cerebrospinal fluid flow in the developing Xenopus brain are regulated by foxj1. Cilia. 2013;2(1):12.

40. Werner ME, Mitchell JW, Putzbach W, Bacon E, Kim SK, Mitchell BJ. Radial intercalation is regulated by the Par complex and the microtubule-stabilizing protein CLAMP/Spef1. J Cell Biol. 2014;206(3):367-76.

41. Chung MI, Kwon T, Tu F, Brooks ER, Gupta R, Meyer M, et al. Coordinated genomic control of ciliogenesis and cell movement by RFX2. Elife. 2014;3:e01439.

42. Ma L, Quigley I, Omran H, Kintner C. Multicilin drives centriole biogenesis via E2f proteins. Genes Dev. 2014;28(13):1461-71.

43. Stubbs JL, Vladar EK, Axelrod JD, Kintner C. Multicilin promotes centriole assembly and ciliogenesis during multiciliate cell differentiation. Nat Cell Biol. 2012;14(2):140-7.

44. Brooks ER, Wallingford JB. Multiciliated cells. Curr Biol. 2014;24(19):R973-82.

45. Mitchell B, Jacobs R, Li J, Chien S, Kintner C. A positive feedback mechanism governs the polarity and motion of motile cilia. Nature. 2007;447(7140):97-101.

46. Kim SK, Shindo A, Park TJ, Oh EC, Ghosh S, Gray RS, et al. Planar cell polarity acts through septins to control collective cell movement and ciliogenesis. Science. 2010;329(5997):1337-40.

47. Tan FE, Vladar EK, Ma L, Fuentealba LC, Hoh R, Espinoza FH, et al. Myb promotes centriole amplification and later steps of the multiciliogenesis program. Development. 2013;140(20):4277-86.

48. Marcet B, Chevalier B, Luxardi G, Coraux C, Zaragosi LE, Cibois M, et al. Control of vertebrate multiciliogenesis by miR-449 through direct repression of the Delta/Notch pathway. Nat Cell Biol. 2011;13(6):693-9.

49. Song R, Walentek P, Sponer N, Klimke A, Lee JS, Dixon G, et al. miR-34/449 miRNAs are required for motile ciliogenesis by repressing cp110. Nature. 2014:510(7503):115-20.

50. Vladar EK, Bayly RD, Sangoram AM, Scott MP, Axelrod JD. Microtubules enable the planar cell polarity of airway cilia. Curr Biol. 2012;22(23):2203-12.

51. Hu Q, Milenkovic L, Jin H, Scott MP, Nachury MV, Spiliotis ET, et al. A septin diffusion barrier at the base of the primary cilium maintains ciliary membrane protein distribution. Science. 2010;329(5990):436-9

52. Zhao H, Zhu L, Zhu Y, Cao J, Li S, Huang Q, et al. The Cep63 paralogue Deup1 enables massive de novo centriole biogenesis for vertebrate multiciliogenesis. Nat Cell Biol. 2013;15(12):1434-44.

53. Guirao B, Meunier A, Mortaud S, Aguilar A, Corsi JM, Strehl L, et al. Coupling between hydrodynamic forces and planar cell polarity orients mammalian motile cilia. Nat Cell Biol. 2010;12(4):341-50.

54. Bhattacharya D, Marfo CA, Li D, Lane M, Khokha MK. CRISPR/Cas9: an inexpensive, efficient loss of function tool to screen human disease genes in Xenopus. Dev Biol. 2015;408:196-204.

55. Boskovski MT, Yuan S, Pedersen NB, Goth CK, Makova S, Clausen H, et al. The heterotaxy gene GALNT11 glycosylates Notch to orchestrate cilia type and laterality. Nature. 2013;504(7480):456-9.

56. Zariwala MA, Gee HY, Kurkowiak M, Al-Mutairi DA, Leigh MW, Hurd TW, et al. ZMYND10 is mutated in primary ciliary dyskinesia and interacts with LRRC6. Am J Hum Genet. 2013;93(2):336-45.

57. Peshkin L, Wühr M, Pearl E, Haas W, Freeman Jr Robert M, Gerhart John C, et al. On the relationship of protein and mRNA dynamics in vertebrate embryonic development. Dev Cell. 2015;35(3):383-94. 